\title{
Capabilities of two- and three-dimensional FDG-PET for detecting small lesions and lymph nodes in the upper torso: a dynamic phantom study
}

\author{
Raymond R. Raylman1, Paul V. Kison², Richard L. Wahl² \\ 1 Department of Radiology, West Virginia University School of Medicine, USA \\ 2 Division of Nuclear Medicine, Department of Internal Medicine, University of Michigan School of Medicine, USA
}

Received 9 July and in revised form 7 September 1998

\begin{abstract}
The capabilities and limitations of two-(2D) and three-dimensional (3D) fluorine-18 fluorodeoxyglucose positron emission tomography (FDG-PET) in detecting small tumors and lymph nodes were studied in a phantom modeling the human chest and axilla. Multiple dual-radionuclide phantom studies were performed. Five hollow spheres ranging in diameter from $3 \mathrm{~mm}$ to $15 \mathrm{~mm}$ were filled with carbon-11 and placed in the axillary and mediastinal regions of an anthropomorphic phantom containing hollow organs filled with ${ }^{18} \mathrm{~F}$ to simulate FDG uptake $1 \mathrm{~h}$ after injection. Dynamic imaging was performed to acquire PET images with varying target-to-background ratios. Imaging was performed in 2D and $3 \mathrm{D}$ acquisition modes, with and without attenuation correction, on a modern PET scanner. Lesion detectability was visually and quantitatively assessed. For objects larger than $9 \mathrm{~mm}$ in diameter, target-to-background ratios ranging from $\sim 3: 1$ to $\sim 10: 1$ were detectable. Objects $<9 \mathrm{~mm}$ in diameter required a target-to-background ratio of $\geq 18: 1$. Target-to-background ratios required for lesion detectability were equivalent for $2 \mathrm{D}$ and $3 \mathrm{D}$ PET images with and without attenuation correction. In conclusion, 2D and 3D PET with attenuation correction consistently detected "tumors" $\geq 9 \mathrm{~mm}$. Lesions $<9 \mathrm{~mm}$ could be detected if there was high enough tumor uptake. No statistically significant differences in lesion detection were found for $2 \mathrm{D}$ versus $3 \mathrm{D}$ PET, or for attenuation-corrected versus non-attenuation-corrected images.
\end{abstract}

Key words: Positron emission tomography - Tumor detection - Nuclear medicine

Eur J Nucl Med (1999) 26:39-45

Correspondence to: R.R. Raylman, Health Sciences Center South, West Virginia University, Radiology/PET Box 9236, Morgantown, WV 26506-9236, USA

\section{Introduction}

The existence and extent of metastatic disease in the lymphatic system is a critical factor in determining the treatment for a patient with cancer. Perhaps the most common noninvasive method for determining the likelihood of metastatic disease in lymph nodes is the use of $\mathrm{x}$-ray computed tomography $(\mathrm{CT})$ or magnetic resonance imaging (MRI) to detect enlarged lymph nodes, which are often believed to be indicative of cancer infiltration. Unfortunately, enlarged lymph nodes may also be caused by inflammation secondary to infection, while tumor cells are often present in normal-sized lymph nodes [1-3]. Therefore, application of anatomical imaging modalities (such as CT and MRI) may present difficulties when these modalities are utilized to differentiate infiltrated from normal nodes. For example, Yokoi et al. found that in the staging of mediastinal lymph nodes in lung cancer patients, CT had a sensitivity of $62 \%$ and a specificity of $80 \%$ [4]. In the staging of lymph nodes in the axillae of patients with breast carcinoma, March et al. found that CT had a sensitivity of $50 \%$, a specificity of $75 \%$, a positive predictive value of $89 \%$ and a negative predictive value of 20\% [5]. Based primarily on the low negative predictive value, they concluded that $\mathrm{CT}$ is not an accurate predictor of axillary lymph node involvement in breast cancer. In a study by Vogel et al., the diameter of 162 lymph nodes from 83 lung cancer patients was measured postoperatively [6]. They showed that $35.7 \%$ of the nodes greater than $20 \mathrm{~mm}$ in diameter (a size that would have resulted in an interpretation as abnormal from CT scans) did not contain cancer. In subjects with lung cancer McCloud et al. found that only 35.9\% of mediastinal lymph nodes greater than $10 \mathrm{~mm}$ in short-axis diameter contained metastatic disease [7]. MRI has been demonstrated to be approximately as accurate as CT in detecting metastatic spread to the lymphatic system. For example, Zerhouni et al. compared CT and MRI for the determination of nodal infiltration in colorectal cancer [8]. The results showed that, in this application, the accuracies of the two methods were equivalent. 
Imaging of cancer using the positron-emitting glucose analog fluorine-18 fluorodeoxyglucose with positron emission tomography (FDG-PET) has been demonstrated in a number of studies to be very accurate, compared with anatomic imaging methods, for the detection of several types of cancer ranging from breast to lung carcinomas [9-14]. While inflammatory responses in lymph nodes can cause false-positive results [15-20], the findings from human studies indicate that the high target-to-background ratio of FDG makes the detection sensitivity and specificity of FDG-PET very good in relatively large (>10 $\mathrm{mm}$ diameter) tumors and lymph nodes. For smaller tumors, however, much of the good biological target-to-background contrast with normal tissues is reduced by resolution effects. This phenomenon affects the ability of FDG-PET to detect tumor-infiltrated lymph nodes, which may be small $(<10 \mathrm{~mm}$ in diameter).

Several studies have reported that FDG-PET is superior to $\mathrm{CT}$ and MRI for the detection of tumor-infiltrated lymph nodes [21-24]. For example, Laubenbacher et al. reported that the sensitivity of FDG-PET for detection of metastatic lymph nodes is $90 \%$ and the specificity is $96 \%$ for patients with neck squamous cell carcinomas, compared with $78 \%$ and $71 \%$, respectively, for MRI in the same patient population [21]. Additionally, in patients with squamous cell carcinoma of the head and neck, Braams et al. found FDG-PET to have a sensitivity of $91 \%$ and a specificity of $88 \%$ [22]. The smallest infiltrated lymph node detected was $4 \mathrm{~mm}$ in diameter. Crippa et al. reported an overall sensitivity, specificity and accuracy of FDG-PET in the detection of axillary lymph nodes in breast cancer of $84 \%, 85 \%$ and $84 \%$, respectively [25]. In esophageal cancer, Luketich et al. reported a sensitivity of $45 \%$, a specificity of $100 \%$, and an accuracy of $48 \%$ for FDG-PET [26]. The relatively low sensitivity and accuracy were due to false-negative results for a number of small tumor-infiltrated lymph nodes, with a mean diameter of $5.2 \mathrm{~mm}$ (range, 2-10 mm). More recently, Crippa et al. reported a good overall diagnostic accuracy of $86 \%$ in the detection of axillary metastases [27] in 27 patients with breast cancer. Higashi et al. found that in a small number of subjects with mediastinal metastases from lung cancer $(n=4)$, FDG-PET was only able to correctly evaluate two of four positive nodes [28]. The two missed tumorinvolved lymph nodes were less than $10 \mathrm{~mm}$ in diameter.

The ability of PET to detect small photon-emitting structures is limited by its finite resolution. The capability of a PET scanner to detect small tumors or infiltrated lymph nodes is hindered by the fact that, for structures less than twice the reconstructed image resolution, the true amount of activity is not completely recovered. This phenomenon, known as the partial volume effect [29], reduces the apparent target-to-background ratio in the reconstructed images. Thus, much of the advantage of the excellent uptake achieved by FDG is, at least partial- ly, lost. As a result, small malignant lesions and tumorinfiltrated lymph nodes may go undetected. The fraction of true activity concentration in an object recovered in PET images can be gauged by a parameter called the recovery coefficient (RC). Kessler et al. examined the detectability of focal areas of tracer uptake with PET in uniform background and derived an expression for the $\mathrm{RC}$ of spherical objects [30]:

$\mathrm{RC}=\operatorname{erf}\left(\frac{R}{\sqrt{2 \sigma}}\right)-\operatorname{erf}\left(\frac{-R}{\sqrt{2 \sigma}}\right)-\frac{1}{\sqrt{2 \pi}} *\left(\frac{R}{\sigma}\right) * e^{-\frac{1}{2} *\left(\frac{R}{\sigma}\right)^{2}}$,

where $R$ is the radius of sphere, $\sigma$ is the composite (average) FWHM for the specific filter and frequency cut-off, and erf represents the error function defined by:

$\operatorname{erf}(x)=\frac{2}{\sqrt{\pi}} \int_{-x}^{x} e^{-t^{2}} d t$.

Most of the FDG-PET studies assessing PET's ability to detect infiltrated lymph nodes were performed using two-dimensional (2D) data acquisitions and human subjects. The advent of three-dimensional (3D) data acquisition hardware and reconstruction software has significantly increased the detection sensitivity of PET scanners compared with the conventional 2D systems (sensitivity to scattered and random events is also increased). Although the use of human subjects demonstrated the feasibility of using PET to detect infiltrated lymph nodes, it did not allow the investigators to fully explore the limitations of the method. For example, what is the minimum size and target-to-background ratio of a lymph node detectable with FDG-PET? How are these limitations affected by lesion and node position in the body? Is attenuation correction necessary for maximum lesion detection? And, how effective is 3D FDG-PET in detecting small objects in the chest compared with 2D? These questions must be addressed so that we may further define the role of FDG-PET in evaluating the extent of disease in cancer patients. In this investigation we explored these questions utilizing a dynamic anthropomorphic torso phantom.

\section{Materials and methods}

To thoroughly study the limitations of FDG-PET in detecting cancer in the upper thorax, a series of tightly controlled experiments using an anthropomorphic torso phantom (Data Spectrum Inc., Chapel Hill, N.C.) were performed. The anthropomorphic torso phantom contained cavities which, once filled with the appropriate amounts of ${ }^{18} \mathrm{~F}$, simulated the FDG concentration in the major organs of the upper human thorax (heart, lung and liver) of a 70$\mathrm{kg}$ subject $1 \mathrm{~h}$ after injection of FDG. Perhaps the most significant advantage of utilizing a phantom is that a multitude of well-defined lesion sizes, number, and locations can be efficiently examined in a controlled fashion.

All data (2D and 3D) were acquired with a General Electric Advance PET scanner [31, 32]. Two-dimensional and 3D scanning sessions were performed on separate days. Images were reconstructed using filtered backprojection into a $256 \times 256$ matrix 
using Ramp and Hanning filters with frequency cutoff values of 0.5 and 0.29 of the Nyquist frequency $(0.5 / \mathrm{mm})$. Scatter correction was applied to all attenuation-corrected data sets.

Scanner resolution Since the partial volume effect is intimately related to the reconstructed resolution of the scanner (Eq. 1), it was necessary to measure the resolution for the filters and cutoff frequencies used in the study. Capillary tubes $(\mathrm{OD}=1.14 \mathrm{~mm}$ ) were filled with ${ }^{18} \mathrm{~F}$ and placed in the "mediastinum" and "left axilla" of the phantom. Additionally, several tubes were positioned transaxially in areas adjacent to these axially oriented tubes. The hollow organs and spaces in the phantom were filled with water; no radioactivity was present in these areas. The measurements were made in the anthropomorphic phantom to recreate the scatter and attenuation conditions present in the images used to assess lesion detectability. PET images were acquired for $20 \mathrm{~min}$, resulting in greater than $50 \mathrm{kcounts} / \mathrm{image}$ plane. As mentioned above, the images were reconstructed with Ramp and Hanning filters with cutoff values of 0.5 and 0.29 of the Nyquist frequency. The Hanning filter is a common smoothing frequency filter often employed in clinical PET imaging. The Ramp filter is less common in the clinical setting, but its use produces the optimal reconstructed image resolution. The frequency cutoff values are representative of the range of values commonly utilized in clinical imaging. At our institutions a Hanning filter with a frequency cutoff of 0.29 the Nyquist frequency is utilized for clinical imaging. Tangential and radial profiles were drawn through the transaxial images of the mediastinal and axillary capillary tubes. The full-width-at-half-maximum (FWHM) of these profiles was measured and reported as the resolution of the scanner at the given filter and cutoff frequency. The transaxial images were reformatted to produce coronal views of the phantom. Profiles were then drawn through the transaxially oriented tubes and the FWHM of these curves used as a measure of axial resolution. Utilizing these measurements, the $\mathrm{RC}$ for each sphere was calculated using Eq. 1.

Sphere detectability In order to investigate the limitations of PET scanner detection of objects in the upper torso, small FDG-avid structures (such as malignant tumors or tumor-infiltrated lymph nodes) were simulated with hollow spheres (diameters of 3, 5, 9, 12 , and $15 \mathrm{~mm}$ ) filled with radionuclide. To explore the relationship amongst object size, target-to-background ratio, and detectability, a series of dual-radionuclide experiments was performed. The purpose of these experiments was to effectively simulate a wide range of contrast ratios between lesion and background (background was defined as the normal tissue immediately adjacent to the lesions), and to determine (for each size object) the minimum ratio necessary for detection by PET.

To simulate the FDG distribution in the normal human thorax $1 \mathrm{~h}$ after administration of $370 \mathrm{MBq}$ of FDG, the hollow organs of the phantom were filled with ${ }^{18} \mathrm{~F}$; as follows: heart, $23.8 \mathrm{kBq} / \mathrm{ml}$; liver, $12.7 \mathrm{kBq} / \mathrm{ml}$; lungs, $3.7 \mathrm{kBq} / \mathrm{ml}$; and adipose tissue, $5.3 \mathrm{kBq} / \mathrm{ml}$ (corresponding to SUVs of $4.5,2.4,0.6$ and 1.0, respectively, for a $70-\mathrm{kg}$ subject). Tracer uptake in the brain was simulated with a 200 -mm-diameter flood phantom, containing an ${ }^{18} \mathrm{~F}$ concentration of $23.7 \mathrm{kBq} / \mathrm{ml}$, placed $180 \mathrm{~mm}$ above the torso phantom on the scanner bed. Bladder uptake was simulated with a beaker containing $500 \mathrm{ml}$ of water and $26.4 \mathrm{MBq}$ of ${ }^{18} \mathrm{~F}$. These values have been previously measured in human subjects [33]. To obtain a wide range of target-to-background ratios, the spheres were filled with high concentrations of ${ }^{11} \mathrm{C}$, which is also a positron emitter, but which has a shorter half-life than ${ }^{18} \mathrm{~F}$ ( 20 min vs $110 \mathrm{~min})$. The difference in radionuclide half-lives between ${ }^{11} \mathrm{C}$ and ${ }^{18} \mathrm{~F}$ produced a range of target-to-background ratios (20:1 to $0.5: 1)$ during the course of a 2 -h data acquisition sequence. These target-to-background ratios span the range of ratios reported in human studies.

A dynamic sequence of 12 PET scans (each 10 min in duration) was acquired. During a majority of the imaging sequence, the absolute radionuclide concentrations in the organs were similar to those present in human studies. One set of spheres was placed in the mediastinal region of the phantom, the other set was positioned in the left axillary region. To explore the effect of photon-attenuation correction on the detectability of spheres, both the $2 \mathrm{D}$ and $3 \mathrm{D}$ data sets were reconstructed with and without attenuation correction. Acquisition of the transmission images using a pair of rotating germanium-68 rod sources was performed immediately following the emission scanning sequence. A total of 300 million true events were acquired. Following the method of Kessler et al. [30] and Links et al. [34], RCs were measured by dividing the maximum pixel value in each sphere by the maximum pixel value in an adjacent background region. These calculations were only performed on attenuation-corrected images.

The sequences of transverse images were viewed by a radiologist highly experienced in evaluating FDG-PET images (R.L.W.) blinded to the positions of the spheres in the phantom. Image sets were randomly shuffled so that only the latest time frames (with the lowest target-to-background ratio) were shown first in order to keep the actual positions of the spheres from the viewer for as long as possible. Each image was scored using a four-point scale $(0=$ normal, $1=$ probably normal, $2=$ probably abnormal and $3=$ definitely abnormal). The mid-time point of the image frame identified as the first where each sphere received a score of 3 (definitely abnormal) was used to calculated the radionuclide concentrations in the spheres and normal areas. These data were then utilized to calculate the minimum target-to-background ratio necessary for detection $[\mathrm{TBRP}(\mathrm{min})]$. This analysis was performed on both $2 \mathrm{D}$ and 3D image sets, with and without attenuation correction and with the four different reconstruction filter-cutoff frequency combinations. The TBRP( $\min )$ values for each test condition and anatomical position were compared using a Student's $t$ test to determine whether there were any statistically significant $(P<0.05)$ differences between 2D and 3D, mediastinal and axillary, and attenuation- and non-attenuation-corrected imaging results. Calculations of the partial volume effect revealed that the smallest object ( $3 \mathrm{~mm}$ in diameter) would be visible only when the target-tobackground ratio was above approximately 120:1. Therefore, the previously described imaging and analysis procedure were repeated with greater target-to-background contrast ratios. The ${ }^{18} \mathrm{~F}$ concentrations in the organs of the phantom were the same as previously described, while the concentration in the spheres was increased to produce ratios of 300:1 to $0.8: 1$ over a 4 -h period. Thus, we were able to track the visibility of the lesions and estimate (for each object size) the minimum target-to-background ratio necessary for detection.

\section{Results}

\section{Scanner resolution and recovery coefficient measurements}

Table 1 shows the measured resolution for each combination of filter and frequency cut off values tested. Figure 1 displays plots of measured RCs versus RCs calculated using Eq. 1. 
A

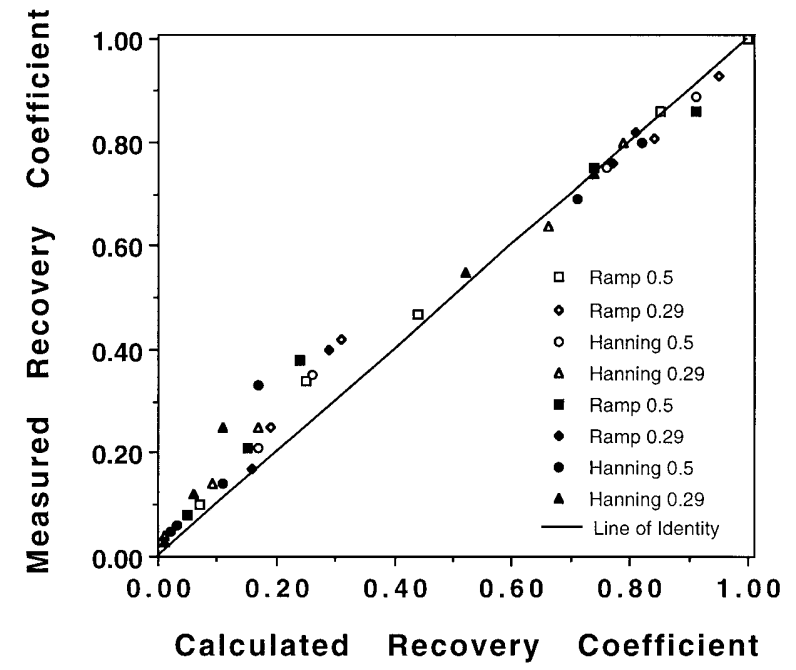

B

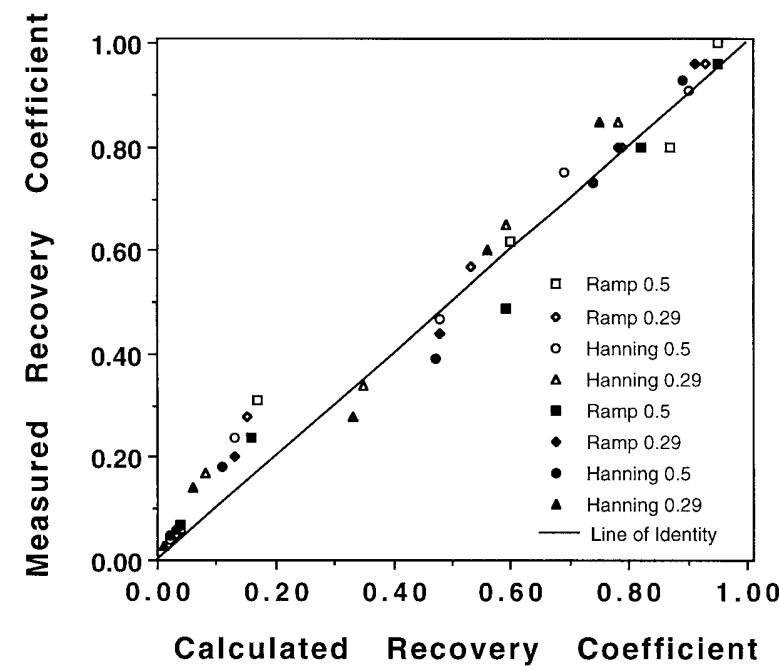

Fig. 1A, B. Plots of measured RCs versus calculated RCs. A Results for the spheres positioned in the mediastinum and axilla measured using a 2D acquisition. B Results for a 3D acquisition

\section{Sphere detectability}

Figure 2 displays representative PET images of the anthropomorphic dynamic phantom. Figure $2 \mathrm{~A}$ shows a transaxial view of the phantom at the level of the 12$\mathrm{mm}$-diameter spheres. Figure 2B shows a coronal view of the phantom at the level of the mediastinum. These
Table 1. Measured scanner 2D and 3D resolutions (FWHM in $\mathrm{mm}$ ) measured for all four of the filter-frequency cutoff combinations utilized in 2D and 3D in the mediastinum and axilla

\begin{tabular}{lclll}
\hline & $\begin{array}{l}\text { Ramp } \\
(0.5)\end{array}$ & $\begin{array}{l}\text { Ramp } \\
(0.29)\end{array}$ & $\begin{array}{l}\text { Hanning } \\
(0.5)\end{array}$ & $\begin{array}{l}\text { Hanning } \\
(0.29)\end{array}$ \\
\hline 2D Mediastinum & & & \\
Radial & 5.3 & 6.3 & 6.2 & 8.5 \\
Tang. & 4.9 & 6.0 & 6.5 & 8.9 \\
Axial & 4.7 & 5.4 & 6.5 & 5.9 \\
2D axilla & & & & \\
Radial & 6.6 & 7.7 & 7.8 & 10.0 \\
Tang. & 4.9 & 5.8 & 61 & 8.7 \\
Axial & 8.0 & 8.0 & 8.1 & 8.2 \\
3D mediastinum & & & \\
Radial & 5.4 & 6.3 & 6.8 & 8.9 \\
Tang. & 5.0 & 5.9 & 6.3 & 8.5 \\
Axial & 8.0 & 8.0 & 8.0 & 8.1 \\
3D axilla & & & & \\
Radial & 5.4 & 6.3 & 6.8 & 8.9 \\
Tang. & 5.2 & 5.9 & 6.4 & 8.8 \\
Axial & 8.4 & 8.5 & 8.4 & 8.4 \\
\hline
\end{tabular}

images were obtained from the first frame and were acquired in 3D and reconstructed with a Hanning filter with a cutoff of 0.29 of the Nyquist frequency. In 2D, the number of total true counts per image plane ranged from $\sim 665 \mid 000$ to $\sim 117 \mid 000$. In $3 \mathrm{D}$, the number of total true counts per plane ranged from $\sim 5$ million to $\sim 1$ million.

Table 2 shows the average values of the minimum target-to-background ratio present in the phantom necessary to detect each sphere [TBRP(min)] when attenuation correction was applied. Table 3 displays the same data for images where no attenuation correction was applied. For each sphere, four ratios were obtained (one for each combination of reconstruction filter and cutoff value); they were combined to produce the average ratios shown in Tables 2 and 3 . The values for the spheres with diameters from $15 \mathrm{~mm}$ to $5 \mathrm{~mm}$ were obtained from the first set of images using the relatively low target-tobackground ratios; results for the 3-mm-diameter sphere

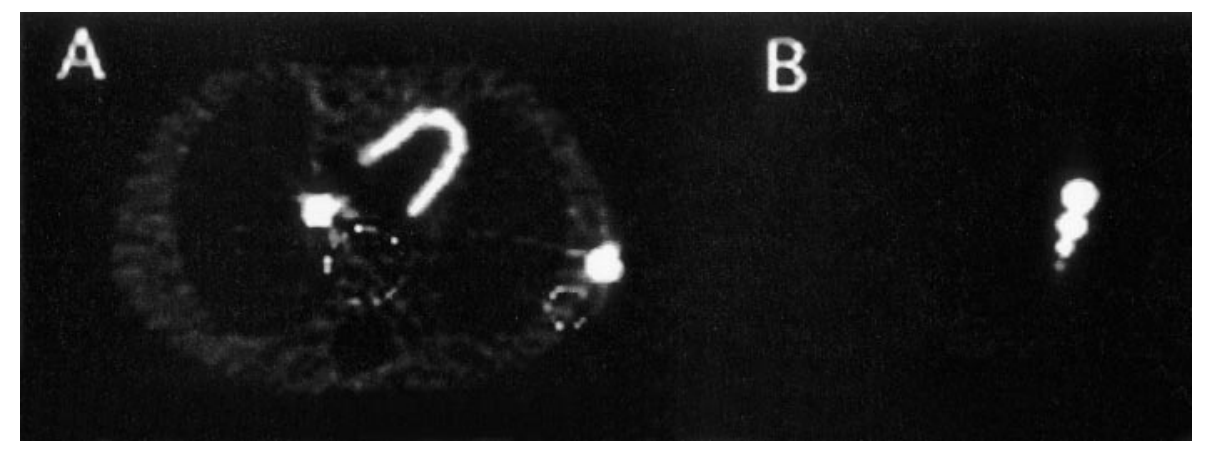

Fig. 2. Representative PET images of the anthropomorphic phantom. A Transaxial view of the 12-mm diameter spheres. B Coronal view of the phantom showing spheres placed in the mediastinum 
Table 2. Minimum target-to-background ratios present in the phantom necessary for detection of the spheres [TBRP(min)] in the axilla and mediastinum for $2 \mathrm{D}$ and $3 \mathrm{D}$ attenuation-corrected images. The values were calculated from all four combinations of filter and frequency cutoffs

\begin{tabular}{lllll}
\hline $\begin{array}{l}\text { Diam. } \\
(\mathrm{mm})\end{array}$ & $\begin{array}{l}\text { 2D } \\
\text { TBRP(min) } \\
\text { Med. }\end{array}$ & $\begin{array}{l}\text { 2D } \\
\text { TBRP(min) } \\
\text { Ax. }\end{array}$ & $\begin{array}{l}\text { 3D } \\
\text { TBRP(min) } \\
\text { Med. }\end{array}$ & $\begin{array}{l}\text { 3D } \\
\text { TBRP(min) } \\
\text { Ax. }\end{array}$ \\
\hline \multirow{2}{*}{15} & 2.8 & 3.2 & 2.7 & 2.7 \\
& $(1.9-3.4)$ & $(2.6-3.4)$ & $(2.2-2.9)$ & $(2.2-2.9)$ \\
12 & 4.2 & 5.9 & 3.9 & 3.9 \\
& $(3.4-4.5)$ & $(3.4-8.0)$ & $(3.9)$ & $(3.9)$ \\
9 & 10.6 & 10.6 & 7.4 & 7.4 \\
& $(10.6)$ & $(10.6)$ & $(6.8-9.0)$ & $(6.8-9.0)$ \\
5 & 18.7 & 18.7 & 16.0 & 18.0 \\
& $(18.7)$ & $(18.7)$ & $(15.2-17.9)$ & $(16.1-18.6)$ \\
3 & 88.1 & 119.6 & 118.3 & 95.3 \\
& $(79.2-91.1)$ & $(115.2-121.3)$ & $(113.5-120.1)$ & $(94.5-96.8)$ \\
\hline
\end{tabular}

Med., Mediastinum; Ax., axilla

Table 3. Average minimum target-to-background ratios present in the phantom necessary for detection of the spheres [TBRP(min)] in the axilla and mediastinum for 2D and 3D images without attenuation correction. The values were calculated from all four combinations of filter and frequency cutoffs

\begin{tabular}{lllll}
\hline $\begin{array}{l}\text { Diam. } \\
(\mathrm{mm})\end{array}$ & $\begin{array}{l}\text { 2D } \\
\text { TBRP(min) } \\
\text { Med. }\end{array}$ & $\begin{array}{l}\text { 2D } \\
\text { TBRP(min) } \\
\text { Ax. }\end{array}$ & $\begin{array}{l}\text { 3D } \\
\text { TBRP(min) } \\
\text { Med. }\end{array}$ & $\begin{array}{l}\text { 3D } \\
\text { TBRP(min) } \\
\text { Ax. }\end{array}$ \\
\hline \multirow{2}{*}{15} & 3.5 & 3.5 & 3.2 & 2.1 \\
& $(2.6-4.5)$ & $(2.6-4.5)$ & $(2.9-3.9)$ & $(1.7-2.2)$ \\
12 & 4.5 & 5.6 & 3.4 & 2.8 \\
& $(4.5)$ & $(4.5-6.0)$ & $(2.9-3.9)$ & $(2.9-3.9)$ \\
9 & 11.4 & 8.7 & 7.3 & 7.9 \\
& $(10.6-14.0)$ & $(8.0-10.6)$ & $(6.8-9.0)$ & $(6.8-9.0)$ \\
5 & 18.7 & 18.7 & 16.0 & 19.2 \\
& $(18.7)$ & $(18.7)$ & $(16.0)$ & $(18.7-20.3)$ \\
3 & 86.6 & 117.3 & 118.0 & 83.5 \\
& $(85.0-88.1)$ & $(115.6-118.0)$ & $(117.2-119.3)$ & $(82.5-85.0)$ \\
\hline
\end{tabular}

Med., Mediastinum; Ax., axilla

were obtained from a second set of high-ratio images. It should be noted that the results for the other size spheres (15 $\mathrm{mm}$ to $5 \mathrm{~mm}$ ) from these high-ratio images, while not given, were consistent with those reported for the low-ratio image sets. Results from the statistical tests of all the data sets revealed no significant differences of $\operatorname{TBRP}(\min )$ s measured for $2 \mathrm{D}$ and $3 \mathrm{D}$, mediastinal and axillary, and attenuation- and non-attenuation-corrected PET images.

\section{Discussion}

While FDG-PET has been demonstrated to be effective in the detection of many cancers and in noninvasively lo- cating the existence of metastatic disease in lymph nodes, this technique is limited by physical constraints. Perhaps the most significant of these constraints is loss of target-to-background contrast due to camera resolution effects. In this study, we explored the limitations of PET in detection of small objects in the human chest imposed by the partial volume effect.

The results in Table 1 demonstrate the spatial dependence of reconstructed resolution. In both $2 \mathrm{D}$ and 3D, the resolution is best in the center (mediastinal region) of the field-of-view (FOV), and gradually degrades towards the edges (axilla) of the FOV. This behavior is due to increased intercrystal scatter at the edges, demonstrated by the sharp increase in the FWHM of the radial component of the line spread function. Use of the Ramp filter produced the best resolution, as did the highest frequency cutoff values. The recovery coefficients shown in Fig. 1 are directly affected by resolution and object size (as seen in Eq. 2). For larger diameter spheres (greater than $9 \mathrm{~mm}$ in diameter, which yield RCs greater than 0.3 ), the measured and calculated RCs are in very good agreement, indicated by the clustering of these points around the line of identity. For smaller spheres the measured values were consistently higher than the calculated values. Links et al. have also observed this phenomenon and attributed it to the increased effect of "spill in" to small spheres from background regions [34]. Therefore, detectability of small spheres may be slightly better than predicted from calculations.

Loss of image contrast of small objects due to resolution effects can be offset by increased target-to-background ratios and increased absolute quantities of tracer in the structures imaged. This phenomenon is demonstrated by the TBRP(min) data presented in Table 2 . Even the smallest 3-mm-diameter sphere is detectable in the PET images at TBRP(min)s greater than 100:1. In 2D PET, the minimum target-to-background concentration ratios necessary for detection of lesions in the mediastinum and the axilla are approximately the same. This finding is somewhat surprising, given the differences in resolution measured in these two regions. Most likely the absence of surrounding organs in the axilla produces better lesion contrast in this region compared with the mediastinum. FDG-avid organs such as the heart and liver are potential sources of scattered activity which can reduce detected target-to-background concentration ratios.

From these data, it is also apparent that objects greater than or equal to $9 \mathrm{~mm}$ in diameter should be detectable in many FDG-PET studies due to the excellent uptake of this tracer. For example, Minn et al. measured a 16:1 target-to-lung ratio for lung tumors imaged with FDG-PET [35]. Objects which are $5 \mathrm{~mm}$ or smaller require much greater tracer concentrations - at least an 18.7:1 ratio -, making it less likely that many lesions or infiltrated lymph nodes in this size range will be detected with current generation PET scanners (assuming that the lesion is not moving during acquisition). These re- 
sults are consistent with anecdotal reports of minimum detectable tumor sizes from human studies [22, 25-28].

In 3D PET with the GE Advance camera, the TBRP(min)s are not significantly different from the 2D results. There is, however, a suggestion in the data that the $3 \mathrm{D}$ values are slightly smaller compared with $2 \mathrm{D}$. This result may be caused by the somewhat better statistical quality of the $3 \mathrm{D}$ images due to the higher number of counts present in these images (detection sensitivity for true events is a factor of $\sim 5.5$ higher in the $3 \mathrm{D}$ acquisition mode compared with the 2D mode). Further study is required to definitively determine the existence and magnitude of this effect. The similarity between data acquired from 2D and 3D images indicates that the increased sensitivity for out-of-scanner activity (such as in the bladder) exhibited by 3D-configured PET scanners does not significantly handicap lesion detection with FDG-PET, at least in the mediastinum and axilla. As with the $2 \mathrm{D}$ results, there is no statistically significant difference between the TBRP(min)s in mediastinum and axilla in 3D.

When photon-attenuation correction is not applied, the appearance of the spheres in the images is altered. As observed by Zasadny et al. [36], the anteroposterior dimensions of the spheres were enlarged and the left-toright dimensions were contracted compared with the attenuation-corrected images. These differences were most evident in the mediastinal region, where the effect of attenuation is greatest due to the depth of the objects. It is also important to note that without an attenuation map, the software of the GE Advance system does not allow scatter correction. Therefore, the images without attenuation correction also did not have scatter correction applied. As the data in Table 3 show, the slight distortions in the apparent shape of the spheres and lack of scatter correction did not significantly change the values of TBRP(min) in the mediastinum compared with the attenuation-corrected results (Table 2). Similarly, the values for the axilla were virtually unchanged between the two sets of data (attenuation-corrected and non-attenuation-corrected). The factors which influence the detectability of small objects in the chest (object size relative to reconstructed resolution and target-to-background ratio) are physical factors virtually unrelated to the type of PET scanner utilized. Thus, although these findings were obtained with a specific model of a widely used PET scanner (GE Advance), the results regarding lesion and lymph node detection in the chest are applicable, at least in a general way, to studies performed with most of the current generation of PET systems.

It must be noted that some uncertainty exists in the determination of TBRP(min)s. Due to the finite length of each frame and the 20-min half-life of ${ }^{11} \mathrm{C}$, sampling of the range of TBRPs was coarse (especially in the early stages of the dynamic sequence). Hence, there may be some uncertainty in determinations of TBRP(min), especially for the smaller spheres. During the course of each 10 min frame the target-to-background ratio changed by approximately $25 \%$. The change from the start of the frame to the mid-time point where the TBRP(min)s were calculated was $13.3 \%$. In addition some human factors could have contributed to error. After several image sets had been read, the once-blinded observer became aware of the likely positions of the spheres in subsequent images. And, even though the viewed image sets were shuffled randomly (as previously described), the partial unblinding of the observer was inevitable. Thus, some bias could be present in the image analysis results, which could have shifted the TBRP(min)s to lower values. Our experimental design, however, attempted to minimize these effects. Furthermore, the sensitivity and resolution of PET scanners in both 2D and 3D modes are functions of axial position (as well as transaxial position). The precise axial locations of the spheres were not known. Therefore, some variations from the reported values of calculated RCs and TBRP(min)s are possible. This variability is less in 3D PET than in 2D PET owing to the greater axial sampling density in 3D PET acquisitions. This unknown positioning is consistent with clinical practice.

In summary, we have investigated the capabilities of 2D and 3D FDG-PET scanning for detecting small tracer-avid objects in the chest and axilla and confirmed that, overall, the ability to detect lesions or lymph nodes greater than $9 \mathrm{~mm}$ in diameter is very good. Target-tobackground ratios needed for detection of objects in this size range are usually attainable in vivo with FDG. Smaller lesions require significantly greater target-tobackground uptake which may be attainable only infrequently with FDG. For attenuation-corrected images, the minimum target-to-background concentration ratios required in both 2D and 3D FDG-PET for detection with the GE Advance PET scanner were approximately the same for the mediastinum and axilla. Similarly, for nonattenuation-corrected images, the required target-tobackground ratios for detection were not significantly different in 2D versus 3D. Due to the limited resolution of the current generation of PET cameras, detection of micrometastatic disease is not currently feasible with PET.

Acknowledgements. We thank Mr. Louis Tluzcek and Dr. Scott Snyder of the University of Michigan PET facility radiochemistry group, and Mr. Bryan Smith and Dr. Samuel Mazza of the West Virginia University PET facility radiochemistry group for the radionuclides utilized in this study. This research was supported by a WVU School of Medicine Research Grant and by a grant from the National Institutes of Health (CA52880).

\section{References}

1. Arita T, Kuramitsu T, Kawamura M, et al. Bronchogenic carcinoma: incidence of metastases to normal sized lymph nodes. Thorax 1995; 50: 1267-1269.

2. Gross BH, Glatzer GM, Orringer MB, Spizarny DL, Flint A. Bronchogenic carcinoma metastatic to normal-sized lymph nodes: frequency and significance. Radiology 1988; 166: 71-74. 
3. Castellino R, Hoppe R, Blank N, et al. Computed tomography, lymphography, and staging laparotomy: correlations in initial staging of Hodgkin's disease. AJR 1984; 143: 37-41.

4. Yokoi K, Okuyama A, Mori D, et al. Mediastinal lymph node metastasis from lung cancer: evaluation with Tl-201 SPECT comparison with CT. Radiology 1994; 192: 813-817.

5. March DE, Wechsler RJ, Kurtz AB, Rosenberg AL, Needleman L. CT-pathologic correlation of axillary lymph nodes in breast carcinoma. J Comput Assist Tomogr 1991;15:440-444.

6. Vogel P, Daschner H, Lenz J, Schafer R. Correlation of lymph node size and metastatic involvement of lymph nodes in bronchial cancer [abstract] Langenbecks Arch Chir 1990; 375: 141-144.

7. McCloud TC, Bourgouin PM, Greenberg RW, Kosiuk JP, Templeton PA, Shepard JAO. Bronchogenic carcinoma: analysis of staging in the mediastinum with $\mathrm{CT}$ by correlative lymph node mapping and sampling. Radiology 1992; 182: 319-323.

8. Zerhouni EA, Rutter C, Hamilton SR, et al. CT and MR imaging in the staging of colorectal carcinoma: report of the Radiology Diagnostic Oncology Group II. Radiology 1996; 200: 443-452.

9. Duhaylongsod FG, Lowe VJ, Patz EF Jr, Vaughn AL, Coleman RE, Wolfe WG. Lung tumor growth correlates with glucose metabolism measured by fluoride- 18 fluorodeoxyglucose positron emission tomography. Ann Thorac Surg 1995; 60: $1348-1352$.

10. Niewig OE, Kim EE, Wong W-H, et al. Positron emission tomography with fluorine-18 deoxyglucose in the detection and staging of breast cancer. Cancer 1993; 71: 3920-3925.

11. Sazon DA, Santiago SM, Soo Hoo GW, et al. Fluorodeoxyglucose-positron emission tomography in the detection and staging of lung cancer. Am J Respir Crit Care Med 1996; 153: 417-421.

12. Tse NY, Hoh CK, Hawkins RA, et al. The application of positron emission tomography imaging with fluoro-deoxyglucose to the evaluation of breast disease. Ann Surg 1992; 216: $27-34$.

13. Valk PE, Pounds TR, Hopkins DM, et al. Staging of non-small cell lung cancer by whole-body positron emission tomographic imaging. Ann Thorac Surg 1995; 60: 1573-1581.

14. Wahl RL, Cody RL, Hutchins GD, Mudgett EE. Primary and metastatic breast carcinoma: initial clinical evaluation with PET with radiolabeled glucose analogue 2-[F-18]-fluoro-2-deoxy-D-glucose. Radiology 1991; 179: 765-770.

15. Kubota R, Yamada S, Ishiwata K, Tamahashi N, Ido T. Intramural distribution of FDG in vivo: high accumulation in macrophages and granulation tissue studied by microautoradiography. J Nucl Med 1992; 33: 1972-1980.

16. Wahl R, Quint LE, Greenough RL, Meyer CR, White RI, Orringer MB. Staging of mediastinal non-small cell lung cancer with FDG-PET, CT and fusion images: preliminary prospective evaluation. Radiology 1994; 191: 371-377.

17. Patz EF, Lowe VJ, Hoffman JM, et al. Focal pulmonary abnormalities: evaluation with F-18 fluorodeoxyglucose PET scanning. Radiology 1993; 188: 487-490.

18. Dewan NA, Gupta NC, Redpenning LS, Phalen JJ, Frick MP. Diagnostic efficacy of PET-FDG imaging in solitary pulmonary nodules: potential role in evaluation and management. Chest 1993; 104: 997-1002.

19. Scott WJ, Schwabe JL, Gupta NC, et al. Positron emission tomography of lung tumors and mediastinal lymph nodes using [18F]fluorodeoxyglucose. Ann Thorac Surg 1994; 58: 698-703.
20. Lowe VJ, Hoffman JM, DeLong DM, Patz EF, Coleman RE. Semiquantitative and visual analysis of FDG-PET images in pulmonary abnormalities. J Nucl Med 1994; 35: 1771-1776.

21. Laubenbacher C, Saumweber D, Wagner-Manslau C, et al. Comparison of fluorine-18-fluorodeoxyglucose PET, MRI and endoscopy for staging head and neck squamous-cell carcinomas. J Nucl Med 1995; 36: 1747-1757.

22. Braams JW, Pruim J, Freling NJ, et al. Detection of lymph node metastases of squamous-cell cancer of the head and neck with FDG-PET and MRI. J Nucl Med 1995; 36: 211-216.

23. Sasaki M, Ichiya Y, Kuwabara Y, et al. The usefulness of FDG positron emission tomography for the detection of mediastinal lymph node metastases in patients with non-small cell lung cancer: a comparative study with $\mathrm{x}$-ray computed tomography. Eur J Nucl Med 1996; 23: 741-747.

24. Scott WJ, Gobar LS, Terry JD, Dewan NA, Sunderland JJ. Mediastinal lymph node staging of non-small-cell lung cancer: a prospective comparison of computed tomography and positron emission tomography. J Thorac Cardiovasc Surg 1996; 111: 642-648.

25. Crippa F, Agresti R, Delle Donne V, et al. The contribution of positron emission tomography (PET) with ${ }^{18} \mathrm{~F}$-Fluorodeoxyglucose (FDG) in the preoperative detection of axillary metastases of breast cancer: the experience of the national cancer institute of milan. Tumori 1997; 83: 542-543.

26. Luketich JD, Schauer PR, Meltzer CC, et al. Role of positron emission tomography in staging esophageal cancer. Ann Thorac Surg 1997; 64: 765-769.

27. Crippa F, Agresti R, Seregni E, et al. Prospective evaluation of fluorine-18-FDG PET in presurgical staging of the axilla in breast cancer. $J$ Nucl Med 1998; 39: 4-8.

28. Higashi K, Nishikawa T, Seki $\mathrm{H}$, et al. Comparison of fluorine-8 FDG PET and Thallium-201 SPECT in evaluation of lung cancer. J Nucl Med 1998; 39: 9-14.

29. Hoffman EJ, Huang SC, Phelps ME. Quantitation in positron emission computed tomography: effect of object size. J Comput Assist Tomogr 1979; 3: 299-308.

30. Kessler RM, Ellis JR Jr, Eden M. Analysis of emission tomographic scan data: limitations imposed by resolution and background. J Comput Assist Tomogr 1984; 8: 514-522.

31. Lewellen TK, Kohlmeyer SG, Miyaoka RS, Schubert SF, Stearns CW. Investigation of the count rate performance of General Electric Advance positron emission tomograph. IEEE Trans Nucl Sci 1995; 42: 1051-1057.

32. Lewellen TK, Kohlmeyer SG, Miyaoka RS, Kaplan MS, Stearns CW, Schubert SF. Investigation of the performance of the General Electric Advance positron emission tomograph in 3D mode. IEEE Trans Nucl Sci 1996; 43: 2199-2206.

33. Zasadny KR, Wahl RL. Standardized uptake values of normal tissues at PET with 2[fluorine-18]-fluoro-2-deoxy-D-glucose: variation with body weight and a method for correction. Radiology 1993; 189: 847-850.

34. Links JM, Zubieta JK, Meltzer CC, Stumpf MJ, Frost JJ. Influence of spatially heterogeneous background activity on "hot object" quantitation in brain emission computed tomography. J Comput Assist Tomogr 1996; 20: 680-687.

35. Minn H, Zasadny KR, Quint LE, Wahl RL. Lung cancer: reproducibility of quantitative measurements for evaluating 2[F-18]-fluoro-2-deoxy-D-glucose uptake at PET. Radiology 1995; 196: 167-173.

36. Zasadny KR, Kison PV, Quint LE, Wahl RL. Untreated lung cancer: quantification of systematic distortion of tumor size and shape in non-attenuation-corrected 2-[fluorine-18]fluoro2-deoxy-D-glucose PET scans. Radiology 1997; 204: 270-283. 